\title{
[WHAT DOCTOR IS / WHAT DOCTOR IS NOT]
}

What Doctor is

a mark or a branding, when a noun,

to sharpen or question, when a verb;

a letter of the alphabet,

OR the entire alphabet;

a physician—without degree or credential—of happening,

a lover of wild creatures,

the foreground, in bright, surgical daylight,

178

a deliveryman, an empty flour sack folded over his head, his Depend box in tow, beat-up Oxfords salvaged from the street, without their laces, spray-painted a cracking-white,

a way in which things are

What Doctor is not

a Greek,

a dandy,

a gesture, expressing High-Minded Ideas 